\title{
Freistellung von Abgeordneten für Betreuungszwecke. Zeitgemäße Neuerung oder eine verpönte Form des Mandats auf Zeit?
}

\author{
Peter Bußjäger
}

\section{Politik als Beruf und seine Schwierigkeiten}

Die Tätigkeit von Politikern in den Parlamenten Deutschlands, Österreichs und der Schweiz wird entweder hauptberuflich oder als einer von mehreren Berufen ausgeübt. Während auf der nationalen Ebene der Anteil der Berufspolitiker deutlich höher ist, üben Parlamentarier auf der Landes- beziehungsweise Kantonsebene neben ihrer politischen Tätigkeit häufig noch einen weiteren Beruf aus. Ihre vor allem in Kumulation mit einem anderen Erwerb insgesamt hohe Arbeitsbelastung tritt zwangsläufig mit familiären Verpflichtungen wie etwa der Kinderbetreuung in Konflikt. Solange sich die Parlamente zu einem guten Teil aus Männern zusammensetzten, wurde dieses Problem eher in den Hintergrund gedrängt und die Wahrnehmung familiärer Pflichten den Partnerinnen der Politiker überlassen. Die öffentliche Wahrnehmung des Themas Vereinbarkeit des Berufes „Politik“ mit Familienpflichten ist mit der Erhöhung des Frauenanteils in den Parlamenten zwar allmählich im Steigen begriffen, insgesamt aber noch bescheiden. ${ }^{1}$ Dies dürfte nicht zuletzt auf das immer noch recht hohe Durchschnittsalter der Abgeordneten zurückzuführen sein. Außerdem sollte nicht unterschätzt werden, dass die Logik der Wiederaufstellung es Abgeordneten nicht ratsam erscheinen lässt, auch nur zeitweise aus der Politik auszuscheiden.

Die Rechtsgrundlagen der politischen Arbeit statten die Abgeordneten zwar mit verschiedenen, vor allem bezügerechtlichen Ansprüchen aus, die es ihnen ermöglichen, Politik als Beruf auszuüben. Bemerkenswerterweise enthalten diese aber im Gegensatz zu anderen arbeitsrechtlichen Bestimmungen keine Regeln wie Mutterschutz oder die Möglichkeit der Karenzierung für Zwecke der Kinderbetreuung. Abgeordnete sind bekanntlich keine Arbeitnehmer im Sinne des Arbeitsrechtes. Sie sind weder an Weisungen noch Aufträge gebunden. Daher schulden sie rechtlich gesehen keine Arbeitsleistung, sondern nehmen in Unabhängigkeit ihr Mandat wahr. Politiker sind diesbezüglich selbständig Erwerbstätigen angenähert. Ein nicht zu übersehendes Argument stellt in diesem Zusammenhang die Personalisierung des Wahlrechtes dar: Schließlich ist der oder die konkrete Abgeordnete in das Parlament gewählt und nicht ein eventuelles Ersatzmitglied. Überhaupt wirft ein zeitweiliges Ausscheiden aus dem Parlament zahlreiche Fragen auf. Damit wird jedoch ein Kernproblem der Vereinbarkeit von Politik und Familie angesprochen: Soll Politik ein Beruf sein, in dem die Frage der Vereinbarkeit der individuellen Dispositionsfähigkeit wie bei den selbständig Erwerbstätigen den Betroffenen überlassen bleibt und der keine fördernden rechtlichen Rahmenbedingungen aufweist? Es gibt mittlerweile in Österreich verschiedene Bestimmungen auf Landesebene, die einen neuen Weg gehen und die im vorliegenden Beitrag - als Beispiele des innovativen Föderalismus - vorgestellt werden sollen. Das Konzept des

1 Siehe aber Elisabeth Wolfgruber, Politische Repräsentation auf Länderebene: Die Landtage und ihre Abgeordneten, in: Herbert Dachs / Franz Fallend / Elisabeth Wolfgruber, Länderpolitik. Politische Strukturen und Entscheidungsprozesse in den österreichischen Bundesländern, Wien 1997, S. $73-229$, S. $167 \mathrm{ff}$. 
innovativen Föderalismus betont den Werkstattcharakter des Föderalismus und seine Funktion, die Gesetzgeber der verschiedenen Ebenen zu einem Wechselspiel von innovatorischer und lernender Gesetzgebung anzuregen. ${ }^{2}$

\section{Freistellung von Abgeordneten im deutschsprachigen Raum im Überblick}

Während in Deutschland die Abgeordneten ihre Funktion im Bundestag und in den Landtagen üblicherweise hauptberuflich ausüben ${ }^{3}$, sind insbesondere die Kantonsparlamente der Schweiz, aber auch die Landtage in Österreich sogenannte Milizparlamente. ${ }^{4}$ Dies bedeutet, dass die politische Arbeit im Regelfall neben einer sonstigen beruflichen Tätigkeit ausgeübt wird. Allerdings ergeben sich zwischen den Abgeordneten in der Schweiz und Österreich beachtliche Unterschiede hinsichtlich ihrer Rechtsstellung. Während die Parlamentarier in den Kantonen Sitzungsgelder erhalten, werden die Abgeordneten der Landtage nach dem österreichischen Bezügerecht durch monatliche Bezüge entschädigt. Auch auf der Ebene des Bundes üben die meisten Abgeordneten neben ihrer parlamentarischen Tätigkeit noch einen weiteren Beruf aus. ${ }^{5}$

Grundsätzlich würde die Vermutung nahe liegen, dass das Thema Freistellung für Betreuungszwecke für ein Berufsparlament näher liegt als für ein Milizparlament, in dem sich die politische Tätigkeit im Regelfall als von vornherein weniger zeitintensiv darstellt. Die empirische Realität unterstützt diese Vermutung indessen nicht. In Deutschland hat sich, soweit ersichtlich, die Gesetzgebung bisher weder auf der Bundes- noch der Landesebene explizit mit den Themen Mutterschutz, Elternurlaub für Abgeordnete und ähnlichem auseinandergesetzt. Lediglich im 23. Änderungsgesetz zum Abgeordnetengesetz wurde 2001 festgelegt, dass die Kostenpauschale bei Abwesenheit im Rahmen des Mutterschutzes nicht gekürzt wird.

In Fällen, in denen sich die Frage einer Befreiung von Abgeordneten von der politischen Arbeit stellt, erfolgt auf Wunsch der Betreffenden eine Beurlaubung auf der Grundlage der Geschäftsordnungen der jeweiligen Parlamente ${ }^{6}$ oder - falls diese keine Urlaubsregelungen

2 Vgl. Peter Bußjäger, Wettbewerb, Benchmarking und innovativer Föderalismus im Alpenraum?, in: ders. / Jens Woelk (Hrsg.), Selbständigkeit und Integration im Alpenraum. Streiflichter zu einem komplexen Projekt, Innsbruck 2009, S. 1 - 11, S. 6 ff.

3 Siehe dazu in Gegenüberstellung zur Situation in Österreich Elisabeth Wolfgruber, a.a.O. (Fn. 1), S. $91 \mathrm{f}$.

4 Für Österreich Elisabeth Wolfgruber, a.a.O. (Fn. 1), S. 92, die jedoch 1997 konstatierte, dass 22 Prozent der befragten Landtagsabgeordneten angaben, mit dieser politischen Tätigkeit vollständig ausgelastet zu sein.

5 Siehe dazu für den österreichischen Nationalrat die Liste der gemäß \$9 BezügebegrenzungsBVG offenzulegenden Bezüge auf http://www.parlinkom.gv.at/LI/ZUSDATEIEN/Offenlegung_BEZ_ NR.pdf (Abruf am 28. Januar 2010).

6 Vgl. \$14 GO Deutscher Bundestag: „Urlaub erteilt der Präsident. Urlaub auf unbestimmte Zeit wird nicht erteilt.“; $\$ 75$ GO des Landtags Baden-Württemberg: „Urlaub bis zu vier Wochen erteilt der Präsident, darüber hinaus der Landtag. Urlaub auf unbestimmte Zeit wird nicht erteilt.“; $\$ 14$ Abs. 5 GO des Landtags Rheinland-Pfalz: „Eine Beurlaubung bis zu einem Monat wird vom Präsidenten erteilt, für längere Zeit von Seiten des Landtags.“; vgl. $\$ 2$ Abs. 2 GO der Bremischen Bürgerschaft: „Urlaub für mehr als sechs Wochen ist vom Vorstand zu genehmigen.“; $\$ 47$ GO des Schleswig-Holsteinischen Landtages sieht die Möglichkeit einer Beurlaubung vor, allerdings ohne nähere Angaben über deren Dauer. 
enthalten - durch ein allgemeines Bekanntgeben ${ }^{7}$ einer Verhinderung. Derartige Regelungen stellen allerdings lediglich Hilfskonstruktionen dar. Sie ermöglichen ein zeitweiliges Fernbleiben von der politischen Arbeit, freilich zu Lasten der Fraktion, die in dieser Zeit auf ihr Mitglied verzichten muss. Die Urlaubsregelungen der Geschäftsordnungen stellen zwar relativ flexible Instrumente dar, sind aber gerade auf solche Fälle nicht abgestellt, in denen sich jemand für eine größere Zeitspanne nicht der politischen Arbeit widmen kann und will.

Eine unbezahlte Beurlaubung dürfte nicht zulässig sein, da mit Übernahme des Mandats von Verfassung wegen ein Anspruch auf angemessene Entschädigung entsteht und die Tätigkeit des Abgeordneten kein arbeitsrechtliches Verhältnis ist, wie schon das „Diäten-Urteil“" des deutschen Bundesverfassungsgerichts feststellte. ${ }^{8}$

Eine etwas andere Situation ergibt sich in der Schweiz. Ganz dem ausgeprägten Föderalismus des Landes entsprechend sind auch die Unterschiede zwischen den Kantonsparlamenten ganz beachtlich. So divergiert die Zahl ihrer Sitzungen stark, so dass sich politische Arbeit in entsprechend unterschiedlichem Ausmaß mit familiären Pflichten vereinbaren lässt. Während beispielsweise in St. Gallen lediglich fünf, dafür im Regelfall mehrtägige Sitzungen des Kantonsrats im Jahr stattfinden, hält der wesentlich kleinere Kanton Schaffhausen circa 20 bis 25 halbtägige Sitzungen pro Jahr ab. ${ }^{9}$

Allerdings sieht die überwiegende Zahl der Kantone eine verpflichtende Teilnahme der Mitglieder an Parlamentssitzungen vor. Im Falle einer Verhinderung hat eine frühzeitige Bekanntgabe zu erfolgen ${ }^{10}$, wobei auch bei längerer Abwesenheit nicht unbedingt eine Beurlaubung erforderlich ist. Auswirkungen haben länger andauernde Absenzen vornehmlich für die Fraktion des betreffenden Abgeordneten.

Von starken Unterschieden geprägt sind auch die Regelungen in den einzelnen Kantonen hinsichtlich der Entgeltleistungen für die Sitzungsteilnahme. Während beispielsweise Nidwalden eine Jahrespauschale an die Parlamentsmitglieder leistet und auch bei Sitzungsabsenzen dieser Betrag nicht gekürzt wird, wird in anderen Kantonen jede Sitzungsteilnahme einzeln in Abhängigkeit von der Anwesenheit entlohnt. In St. Gallen, Zug oder Solothurn erhalten die Mitglieder ein Sitzungsgeld, wenn sie an der Sitzung teilnehmen; wenn sie nicht anwesend sind, entfällt die Leistung. ${ }^{11}$ Allerdings sind die Abgeordneten von Solothurn insofern bessergestellt als die Abgeordneten anderer Kantone, als sie im Falle einer erforderlichen Kinderbetreuung für diesen Zeitraum eine Drittperson mit der Betreuung beauftragen können und eine Entschädigung erhalten. ${ }^{12}$ Basel-Stadt ${ }^{13}$ hatte ebenfalls expli-

7 Vgl. $\$ 4$ Abs. 2 GO für den Bayerischen Landtag.

8 BVerfGE 40, 296.

9 Vgl. dazu Stephan Foerster, Das politische System des Kantons Schaffhausen. Akteure, Institutionen und Entscheidungsprozesse in einem Kleingliedstaat, Tübingen 2005, S. 69. Zu St. Gallen: https://www.ratsinfo.sg.ch/t/kantonsrat.sessionen.html (Abruf am 28. Januar 2010).

10 Vgl. etwa $\$ 14$ der GO des Grossen Rates des Kantons Thurgau (RB 171.1).

11 Eigene Erhebungen des Instituts für Föderalismus, Innsbruck.

12 Vgl. $\$ 28,6$ Entschädigungen Kantonsratsgesetz iVm $₫ 38$ Geschäftsreglement des Kantonsrates von Solothurn: „Ratsmitgliedern, die für die Betreuung von bis zu 12-jährigen Kindern oder pflegebedürftigen Angehörigen verantwortlich sind und dafür regelmäßige Auslagen haben, wird auf begründetes Gesuch hin eine Entschädigung von 100 Franken pro Sitzungshalbtag ausgerichtet.“

13 Siehe dazu Grosser Rat des Kantons Basel-Stadt, Bericht des Ratsbüros zur Anpassung der Entschädigungen des Grossen Rates, 10. September 2007, S. 6, www.grosserrat.bs.ch/suche/geschaefte/details/?idurl=07.5236 (Abruf am 28. Januar 2010). 
zit Kinderbetreuungsentschädigungen eingeführt, diese aber aufgrund der pauschalen Vergütung von CHF 4.000 pro Jahr für die Teilnahme an den Sitzungen mittlerweile wieder abgeschafft. Der Kanton Zürich hingegen hat explizit Regelungen für Dienstfreistellungen aufgrund von Kinderbetreuung oder zur Fortbildung getroffen und deckt die Zeit der Abwesenheit aufgrund parlamentarischer Tätigkeit durch die Leistung einer jährlichen Grundentschädigung von CHF 4.000 ab. ${ }^{14}$

Die Bestimmungen in den österreichischen Parlamenten ermöglichen grundsätzlich ebenfalls kurzfristige Beurlaubungen. Sie ähneln weitgehend den in Deutschland getroffenen Bestimmungen. ${ }^{15}$ Spezifische Regelungen für die Freistellung für Betreuungszwecke gibt es mittlerweile in mehreren Ländern (siehe dazu näher unter 3.).

\section{Innovativer Föderalismus: Die Regelung über die Karenzierung von Abgeordneten in den österreichischen Bundesländern}

Die Länder Salzburg, Tirol und Vorarlberg verfügen über Regelungen in den Landesverfassungen, Landtagswahlgesetzen und/oder den Geschäftsordnungen der Landtage, auf deren Basis eine zeitlich befristete Karenzierung zulässig ist. Sie werden im Folgenden entsprechend der chronologischen Folge ihrer Erlassung vorgestellt:

\subsection{Tirol}

In Tirol kann ein Abgeordneter in einer Sitzung durch ein Ersatzmitglied vertreten werden. Diese Regelung ist in Österreich einmalig. Sie findet ihre Grundlage in $\$ 70$ Abs. 1 lit. e) Tiroler Landtagswahlgesetz, wonach für einen Abgeordneten, wenn er für die gesamte Dauer einer Sitzung beurlaubt wird, das jeweils nächste Ersatzmitglied auf dem betreffenden Wahlvorschlag zu berufen ist. Die Gründe für die „Beurlaubung“ eines Abgeordneten werden nicht näher umschrieben. Die Praxis ist eher großzügig und würde auch längere Karenzierungen zur Pflege und Betreuung von Angehörigen akzeptieren.

Das berufene Ersatzmitglied genießt Immunität nur während des Zeitraumes, in dem es zur Teilnahme an der Tätigkeit des Landtages einberufen wird (Art. 32 Abs. 7 Tiroler Landesordnung). Das Ersatzmitglied bleibt nach Rückkehr des Vertretenen von jeder weiteren Tätigkeit im Landtag ausgeschlossen.

Im Falle Tirols handelt es sich somit um eine Art erweiterter Urlaubsregelung mit der Besonderheit, dass das beurlaubte Mitglied des Landtags durch ein Ersatzmitglied vertreten wird. Es handelte sich um die erste Regelung dieser Art in Österreich.

14 Eigene Erhebungen des Instituts für Föderalismus.

15 Vgl. etwa $\$ 17$ GO LT Burgenland; $\$ 6$ GO LT Kärnten (nur Bekanntgabe der Verhinderung, keine Urlaubserteilung); $\$ 7$ GO LT Niederösterreich; $\$ 10$ GO LT Oberösterreich; $\$ 9$ GO LT Steiermark; $\$ 14$ GO LT Wien (nur Bekanntgabe der Verhinderung, keine Urlaubserteilung). 


\subsection{Vorarlberg}

Gemäß Art. 15 Abs. 5 der Vorarlberger Landesverfassung (Vlbg. LV.) ${ }^{16}$ kann seit 2007 durch Gesetz für einzelne, genau bestimmte Gründe eine Karenzierung von Abgeordneten über deren Ersuchen sowie ihre Vertretung in ihrer Funktion für die Dauer von mindestens drei Monaten und höchstens 14 Monaten vorgesehen werden. Diese einzelnen, genau bestimmten Gründe ergeben sich aus dem Landtagswahlgesetz (\$ 66 Landtagswahlgesetz ${ }^{17}$ ). Sie bestehen in der Pflege und Betreuung eines Kindes oder von nahen Angehörigen.

Für den Zeitraum der Karenzierung rückt das nach den Bestimmungen des Landtagswahlgesetzes zu berufende Ersatzmitglied in den Landtag nach. Das Ersatzmitglied wird zum Abgeordneten und nimmt alle Rechte und Pflichten des vertretenen Abgeordneten wahr. Auch bezügerechtlich rückt es an die Stelle des Vertretenen. Das Ersatzmitglied verliert seine Eigenschaft als Abgeordneter mit dem Ablauf der Karenzierung, ohne dass es eines weiteren Aktes bedarf.

Die Karenzierung darf vom Präsidenten des Landtags nur auf Antrag des betreffenden Abgeordneten und höchstens für die beantragte Dauer gewährt werden. Eine Verlängerung ist unzulässig. Als Rechtsform der Karenzierung ist ein Bescheid vorgesehen, was bedeutet, dass der Landtagspräsident in dieser Funktion zu einem obersten Verwaltungsorgan wird.

Die Regelung Vorarlbergs bildete - in einer gewissen Anlehnung an Tirol - offenbar die erste im deutschsprachigen Raum, die sich explizit dem Thema der Freistellung von Abgeordneten als Mutterschutz und für die Betreuung von Kindern sowie sonstiger naher Angehöriger detailliert widmete und spezifische Regelungen für diesen Fall beinhaltete.

\subsection{Salzburg}

Gemäß Art. 32a der Salzburger Landesverfassung (Sbg. LVG) ${ }^{18}$ kann durch Gesetz vorgesehen werden, dass Mitglieder des Landtages aus bestimmten Gründen für die Dauer von höchstens einem Jahr Karenzurlaub gegen Entfall der Bezüge nehmen können. Für diese Zeit wird das Mandat durch einen Bewerber der wahlwerbenden Partei, der das in Karenzurlaub befindliche Mitglied angehört, ausgeübt (Vertreter).

Gemäß $\$ 5$ Abs. 3 des Geschäftsordnungsgesetzes des Salzburger Landtages ${ }^{19}$ können einen Karenzurlaub gegen Entfall der Bezüge in der Dauer von höchstens einem Jahr Mitglieder des Landtages in Anspruch nehmen, wenn sie (a) Mutter oder Vater eines Kindes werden, und zwar ab der Geburt des Kindes; (b) schwer erkrankte Angehörige (\$ 123 ASVG) pflegen. Abgeordnete, die einen solchen Karenzurlaub in Anspruch nehmen wollen, haben dies dem Präsidenten und der Landeswahlbehörde unter Angabe des Zeitraumes des Karenzurlaubs mitzuteilen. Die Landeswahlbehörde hat den Vertreter des in Karenzurlaub befindlichen Mitgliedes unter sinngemäßer Anwendung der $\$ \$ 98$ und 101 der Salzburger Landtagswahlordnung (1998) für die angegebene Zeit des Karenzurlaubs zu berufen und zum Eintritt in den Salzburger Landtag zu legitimieren. Der Vertreter ist unter sinngemäßer Anwendung des $\$ 7$ zu vereidigen.

16 Die vorliegende Bestimmung beruht auf der Fassung LGBl. Nr. 52 / 2007.

17 Die vorliegende Bestimmung beruht auf der Fassung LGBl. Nr. 53 / 2007.

18 Die vorliegende Bestimmung beruht auf der Fassung LGBl. Nr. 38 / 2008.

19 Ebenda. 
Diese Regelung gilt seit dem Jahr 2008. Ihr Vorbild war unverkennbar das Vorarlberger Modell, das von Salzburg im Sinne des lernenden Föderalismus weiterentwickelt beziehungsweise auf die dortigen Verhältnisse angepasst wurde.

\section{Verfassungsrechtliche Implikationen und Schranken der Verfassungsautonomie der österreichischen Länder}

Grundsätzlich sind die österreichischen Länder befugt, im Rahmen ihrer Verfassungsautonomie - „soweit dadurch die Bundesverfassung nicht berührt wird“ (Art. 99 Abs. 1 B-VG) - die Rechtsstellung der Abgeordneten in den Landtagen zu regeln. Judikatur und Lehre interpretieren das Verbot des Nicht-Berührens der Bundesverfassung als ein Verbot, ihren Bestimmungen explizit zu widersprechen, woraus sich ein grundsätzlich weites Feld der Verfassungsautonomie eröffnet. ${ }^{20}$ Es gibt indessen, gerade was die Rechtsstellung der Mandatare betrifft, einige bundesverfassungsrechtliche Restriktionen: (1) Den Ländern ist die Immunität der Abgeordneten (Art. 57 B-VG) bereits bundesverfassungsrechtlich vorgegeben (Art. 96 Abs. 1 B-VG). (2) Nach Art. 96 Abs. 3 B-VG kann durch Landesgesetz für Mitglieder des Landtages, die aus Anlass ihrer Wahl in den Bundesrat ${ }^{21}$ oder in die Landesregierung auf ihr Mandat verzichten, „eine dem Art. 56 Abs. 2 bis 4 B-VG entsprechende Regelung getroffen werden“. Art. 56 Abs. 2 bis 4 B-VG regeln das so genannte „Mandat auf Zeit" für die Abgeordneten des Nationalrats: Danach kann ein Abgeordneter, der auf sein Mandat wegen der Übernahme einer Funktion in der Bundesregierung verzichtet hat $^{22}$, dieses nach dem Ausscheiden aus der genannten Funktion wieder annehmen. ${ }^{23}$ Die Länder dürfen demnach ihrerseits auch den Fall regeln, dass ein Abgeordneter, der zum Mitglied der Landesregierung oder des Bundesrates wurde und auf sein Mandat verzichtet, nach Beendigung der betreffenden Funktion wieder als Abgeordneter in den Landtag zurückkehren kann.

Damit stellt sich die Frage, ob Art. 96 Abs. 3 B-VG den zeitlich befristeten Rückzug aus einer Funktion in einem Landtag abschließend regelt oder eben nur den Fall des „Mandats auf Zeit“, das darin besteht, dass ein Abgeordneter in eine andere politische Funktion berufen wird. Falls ersteres zutreffen würde, wären die oben angeführten Regelungen der Landesverfassungen Vorarlbergs und Salzburgs insoweit bundesverfassungswidrig, als sie einen besonderen Fall eines Mandats auf Zeit regeln würden, der von Art. 96 Abs. 3 B-VG nicht gedeckt wäre. $^{24}$

20 Dazu näher Peter Pernthaler, Österreichisches Bundesstaatsrecht, Wien 2004, S. 459 ff.

21 Die Mitglieder des österreichischen Bundesrates werden gemäß Art. 35 Abs. 1 B-VG von den Landtagen gewählt.

22 Es besteht zwar keine verfassungsrechtliche Unvereinbarkeit zwischen der Mitgliedschaft in der Bundesregierung und dem Mandat im Nationalrat, es ist aber Praxis, dass ein zum Mitglied der Bundesregierung ernannter Abgeordneter sein Mandat zurückgibt. Für diesen Fall schafft der hier behandelte Art. 56 Abs. 2 bis 4 B-VG eine besondere Regelung.

23 In Deutschland hat der Hessische Staatsgerichtshof das nicht näher befristete, jederzeit widerrufliche, einfachgesetzliche „ruhende Mandat“ für verfassungswidrig erklärt, vgl. ESVGH 27, 193 (197 ff.).

24 So auch die Argumentation des Bundeskanzleramtes im Zusammenhang mit dem Erlass der Regelung der Vorarlberger Landesverfassung (BKA-650.058/0004-V/2/2007). 
Die Karenzierung eines Abgeordneten ist mit dem Sachverhalt, den Art. 96 Abs. 3 B-VG in Zusammenhang mit Art. 56 Abs. 2 bis 4 B-VG vor Augen hat, allerdings gar nicht zu vergleichen: Im Fall der Karenzierung verzichtet das Mitglied nicht für eine grundsätzlich unbestimmte Zeit auf sein Mandat, sondern wird für eine zwölf Monate nicht übersteigende Zeit „beurlaubt“ und bleibt Abgeordneter. Das ist ein gewichtiger Unterschied zum „Mandat auf Zeit" und dem mit ihm verbundenen Privileg einer gesicherten Rückkehr zu einem unbestimmten Zeitpunkt.

Außerdem gibt es auch andere Formen eines zeitweiligen Ruhens der Ausübung einer parlamentarischen Funktion, nämlich die „Beurlaubung“, die eine gängige Institution des Parlamentarismus auch in Österreich darstellt. Die Geschäftsordnungen verlangen dazu im Allgemeinen „triftige Gründe“, ohne näher auszuführen, welche dies sein können. ${ }^{25}$ Die Karenzierung ist, wie auch aus dem oben vorgenommenen Rechtsvergleich hervorgeht, eher mit einem solchen Fall vergleichbar.

Dieser Meinung steht die Auffassung Bernd Wiesers gegenüber, der eine zeitliche Beschränkung der Mandatsausübung (für den nachgerückten Abgeordneten) generell mit dem - auch für die Landtage geltenden - Prinzip des freien Mandats für unvereinbar erachtet. ${ }^{26}$ Wieser übersieht allerdings, dass der österreichische Verfassungsgerichtshof bei entsprechender verfassungsrechtlicher Grundlage, die klarerweise auch eine landesverfassungsrechtliche sein kann, eine vorzeitige Abberufung eines Mandatars (in unserem Falle des nachgerückten) für möglich hält. ${ }^{27}$ Auch Wolfgang Pesendorfer ${ }^{28}$ sieht geringen Spielraum der Länder bei der Einführung eines „Mandats auf Zeit“ und hält lediglich solche Regelungen für zulässig, die normativ Art. 56 Abs. 2 bis 4 B-VG für den Bund entsprechen. Allerdings bezieht er sich offenkundig auf Fälle, die in ihrer Art mit den in Art. 96 Abs. 3 B-VG angeführten vergleichbar sind, ohne sich mit anderen Varianten auseinanderzusetzen. ${ }^{29}$ Die Freistellung von Abgeordneten für Betreuungszwecke ist aber gerade kein Mandat auf Zeit im Sinne der bundesverfassungsrechtlichen Bestimmung.

Es ist daher davon auszugehen, dass es im Rahmen der Verfassungsautonomie der österreichischen Länder liegt, Regelungen über die Karenzierung von Abgeordneten, wie sie insbesondere in Salzburg und Vorarlberg verankert wurden, vorzusehen.

\section{Karenzierung von Abgeordneten aus rechtspolitischer Perspektive}

Die in den genannten Beispielsfällen getroffenen Regelungen über die Karenzierung von Abgeordneten bewegen sich in einem Spannungsfeld: Auf der einen Seite gibt es gesellschaftspolitische Notwendigkeiten, nämlich Personen, die Betreuungspflichten wahrnehmen - und dies sind im Regelfall Frauen -, die Betätigung in der Politik zu erleichtern.

25 Vgl. etwa $₫ 17$ GO LT Burgenland; $₫ 6$ GO LT Kärnten; $\$ 10$ GO LT Oberösterreich; $\$ 9$ GO LT Steiermark.

26 Vgl. Bernd Wieser, Kommentar zu Art. 96 B-VG, in: Karl Korinek / Michael Holoubek (Hrsg.), Österreichisches Bundesverfassungsrecht, Loseblattsammlung, Wien 1999, Rn. 2.

27 VfGH, VfSlg $3426 / 1958$.

28 Wolfgang Pesendorfer, Kommentar zu Art. 96 B-VG, in: Heinz Peter Rill / Heinz Schäffer (Hrsg.), Bundesverfassungsrecht, Wien 2002, Rn. 4.

29 Ebenda. 
Auf der anderen Seite steht das Anliegen, der in der politischen Realität schon genug beschädigten personenbezogenen Wahl der Mitglieder der Parlamente zum Durchbruch zu verhelfen. Das „Mandat auf Zeit“, also die auf einen kurzen Zeitraum begrenzte Mitgliedschaft in einem Parlament gleichsam als Platzhalter für den eigentlichen Abgeordneten, ist deshalb rechtspolitisch äußerst umstritten und wird als eine Pervertierung des Gedankens der Verantwortlichkeit des Mandatsträgers gegenüber den Wählern kritisiert. ${ }^{30}$

Verfassungspolitisch ist es allerdings genauso problematisch, die Mitgliedschaft in einem Parlament als Zufälligkeit und Gelegenheitsfunktion zu behandeln, wie die Benachteiligung von Frauen, die Betreuungspflichten wahrnehmen müssen und wollen, als gegeben hinzunehmen. Es ist daher nach einem Interessenausgleich zu suchen, der in einer unwiderruflich befristeten und insgesamt - im Vergleich zur gesamten Dauer der Mitgliedschaft im Parlament - zeitlich untergeordneten Vertretung noch gegeben gelöst erscheint. Eine andere Haltung läuft Gefahr, politische Arbeit als belanglose Nebentätigkeit zu diskreditieren und nicht als einen - mitunter neben anderen Erwerbstätigkeiten ausgeübten - Beruf zu achten.

In der Gewährung von Beurlaubung wie der Freistellung für Zwecke der Betreuung naher Angehöriger wird dem jeweiligen Parlamentspräsidenten eine hoheitliche Funktion übertragen, die an seine Unabhängigkeit und Neutralität gegenüber dem politischen Kräftespiel durchaus hohe Anforderungen stellt. Umso wichtiger ist in diesen Fällen daher eine transparente, sachlich nachvollziehbare Praxis.

\section{Behutsame Regelungen zur Freistellung von Abgeordneten nötig}

Die Freistellung von Abgeordneten auf Zeit für die Ausübung von Betreuungspflichten ist ein wichtiges Instrument, die Vereinbarkeit von Politik, Beruf und Familie zu verbessern. Es ist allerdings eine Abwägung erforderlich mit dem Interesse, die Personenbezogenheit einer Wahl, die in der Parteiendemokratie nur beschränkt gewährleistet ist, nicht noch undeutlicher werden zu lassen. Aus diesem Grund sollten solche Änderungen nur behutsam und unter engen rechtlichen Rahmenbedingungen vorgenommen werden. Eine vollständige Angleichung an die Verhältnisse im Erwerbsleben erscheint nicht angebracht.

Die in Österreich zuerst im Land Vorarlberg, dann in Salzburg geschaffenen Regelungen sind ein Beispiel der Innovationsfähigkeit des Föderalismus. Ob sich die Bestimmungen bewähren werden, lässt sich derzeit nicht prognostizieren, da es noch keine Anwendungsfälle gibt.

30 Vgl. zur rechtspolitischen Diskussion in Österreich Kathrin Stainer-Hämmerle, Das Mandat auf Zeit, Diplomarbeit, Universität Innsbruck 2000. 